Behavioral/Cognitive

\title{
Savings upon Re-Aiming in Visuomotor Adaptation
}

\author{
DJ. Ryan Morehead, ${ }^{1}$ Salman E. Qasim, ${ }^{2}$ Matthew J. Crossley, ${ }^{1}$ and Richard Ivry ${ }^{1}$ \\ ${ }^{1}$ Department of Psychology, University of California, Berkeley, California 94720, and ${ }^{2}$ Department of Neurological Surgery, University of California, \\ San Francisco, California 94143
}

Sensorimotor adaptation has traditionally been viewed as a purely error-based process. There is, however, growing appreciation for the idea that performance changes in these tasks can arise from the interplay of error-based adaptation with other learning processes. The challenge is to specify constraints on these different processes, elucidating their respective contributions to performance, as well as the manner in which they interact. We address this question by exploring constraints on savings, the phenomenon in which people show faster performance gains when the same learning task is repeated. In a series of five experiments, we demonstrate that error-based learning associated with sensorimotor adaptation does not contribute to savings. Instead, savings reflects improvements in action selection, rather than motor execution.

Key words: adaptation; motor learning; reaching; visuomotor

\section{Significance Statement}

Savings is the phenomenon in which people show faster relearning of a previously forgotten memory. In the motor learning domain, this phenomenon has been a puzzle for learning models that operate exclusively on error-based learning processes. We demonstrate, in a series of experiments, that savings selectively reflects improvements in action selection: Participants are more adept in invoking an appropriate aiming strategy when presented with a previously experienced perturbation. Indeed, improvements in action selection appear to be the sole source of savings in visuomotor adaptation tasks. We observe no evidence of savings in implicit error-based adaptation.

\section{Introduction}

Humans do a remarkably good job in compensating for natural and artificial perturbations of the sensorimotor system. This type of motor learning, adaptation, has served as a cornerstone in understanding motor control and motor memory. Studies of adaptation have highlighted an error-based form of learning in which change is driven by the difference between predicted and actual sensory feedback (Wolpert et al., 1995). The core feature of these algorithms is that they minimize sensory prediction error, dynamically moving toward a steady state in which the adapted motor commands will result in the intended sensory outcome.

On the whole, standard motor learning models that respond only to error do a good job of describing behavior in a range of

\footnotetext{
Received March 17, 2015; revised Sept. 9, 2015; accepted Sept. 17, 2015.

Author contributions: J.R.M., M.J.C., and R.I. designed research; J.R.M. and S.E.Q. performed research; J.R.M. analyzed data; J.R.M. and R.I. wrote the paper.

This work was supported by National Institutes of Health Grants NS074917 and NS092079. We thank Joern Diedrichsen for extremely helpful discussions and assistance designing experiments; Jordan Taylor for technical assistance with Experiment 3 and for helpful comments on the manuscript; and Elizabeth Marrone, Shabnam Bonyadi, Chelsea Daniels, and Dashel Thompson for help collecting experimental data.

The authors declare no competing financial interests.

Correspondence should be addressed to Dr. J. Ryan Morehead, School of Engineering and Applied Sciences, Harvard University, 60 0xford Street, Cambridge, MA 02138. E-mail: ryanmorehead@seas.harvard.edu.

DOI:10.1523/JNEUROSCI.1046-15.2015

Copyright $\odot 2015$ the authors $\quad 0270-6474 / 15 / 3514386-11 \$ 15.00 / 0$
}

adaptation tasks. However, these models cannot account for a well-described phenomenon in learning, savings (Krakauer et al., 2005; Zarahn et al., 2008). Savings is defined as faster learning when information is presented a second time after being forgotten, compared with the rate of initial learning (Ebbinghaus, 1913; Krakauer, 2009). This faster relearning has canonically been thought to reflect the existence of a "hidden" trace, or residual representation of the initial learning experience, even if the effects of this experience are no longer manifest. Variants of this idea have been advanced to describe changes in behavior (Ebbinghaus, 1913) or to characterize the functional role of physiological mechanisms of learning, such as the latent facilitation of synaptic potentiation (Medina et al., 2001).

Several classes of error-based models have been proposed to account for savings in sensorimotor adaptation tasks. One class centers on the idea that multiple error-based adaptation mechanisms operate in parallel, but at different rates (Smith et al., 2006; Zarahn et al., 2008). A second posits that people learn to switch between different internal models, one associated with the baseline context and at least one other associated with the perturbation context (Haruno et al., 2001; Lee and Schweighofer, 2009), or that performance reflects the combined output of multiple internal models (Pearson et al., 2010). A third class builds on the context idea but assumes that the learning process is modified by 
the context. For example, the large error encountered when a perturbation is reintroduced may entail the recruitment of additional error-based processes (Berniker and Körding, 2011) or lead to a change in the gain on the learning rate based on the direction and magnitude of past errors (Herzfeld et al., 2014).

These models share the idea that savings arises from faster modification, or latent storage and subsequent recall, of internal models that specify how intended sensory goal states are translated into joint torques. In contrast, recent work has shown that the pattern of behavior observed in visuomotor savings experiments is often inconsistent with an error-based process, and may be better explained as reflecting changes in action selection that facilitate performance (Huang et al., 2011; Haith et al., 2015; Huberdeau et al., 2015; McDougle et al., 2015; Orban de Xivry and Lefévre, 2015).

Behaviorally, it is often difficult to distinguish between performance gains that are due to faster adaptation of an internal model, recall of a strategy, or the combined effects of strategic and adaptation processes. Here, in a series of experiments using a converging set of measures, we test the hypothesis that savings arises from an action selection process rather than error-based adaptation. Specifically, we propose that, when a previously experienced perturbation is re-encountered, participants may achieve savings by recalling an aiming strategy that facilitated performance when participants first encountered the perturbation. Recalling this strategy could allow for rapid improvements in performance, even in the absence of "true" adaptation (recalibration of a sensorimotor mapping).

\section{Materials and Methods}

Participants. A total of 130 participants ( 43 males, age $21 \pm 2$ years) were recruited from the undergraduate population at the University of California, Berkeley through the Department of Psychology research participation pool. All participants were right handed, as measured by the Edinburgh Handedness Inventory (Oldfield, 1971). Participants received either class credit or financial compensation for their participation. The experimental protocol was approved by the institutional review board of the University of California, Berkeley.

Experimental apparatus. Participants reached to visual targets by sliding their hands across a table surface. Kinematic data were obtained via a six-axis magnetic position sensor (miniBird, Ascension Technology) attached to the index finger. A ceiling-mounted LCD projector projected the visual stimuli onto a screen that, through a reflecting mirror, created the illusion that the targets appeared in the same plane as the table surface (Fig. 1a). The experimental task was implemented using custom software written in Python 2.7.

Reaching task. On each trial, the participant reached from a central starting position ( $1.2 \mathrm{~cm}$ diameter circle) to a blue target $(1.6 \mathrm{~cm}$ diameter circle). Targets were always centered at a distance of $10 \mathrm{~cm}$ from the start position but could appear at either one of two positions, $30^{\circ}$ to the left or $30^{\circ}$ to the right of midline, or at one of four target positions positioned $90^{\circ}$ increments around the workspace (Fig. 1b). The target position for a given trial was pseudorandomly selected so that each target was presented 4 times out of every 8 trials (two-target conditions) or twice out of every 8 trials (four-target conditions). The projection screen and mirror precluded vision of the arm. Feedback of the position of the index finger was indicated by a small white cursor $(0.8 \mathrm{~cm}$ diameter). The participant was instructed to "hit the blue target with your white dot, and make sure you move fast." The feedback cursor was visible until movement amplitude exceeded $10 \mathrm{~cm}$. At this point, the cursor froze for $2 \mathrm{~s}$ (Experiments 1, 2, 4, 5) or $0.5 \mathrm{~s}$ (Experiment 3), providing spatial feedback of the accuracy of the reach at the target amplitude. Participants were required to traverse the $10 \mathrm{~cm}$ reach in $<300 \mathrm{~ms}$. If this criterion was exceeded, the computer played an auditory message, "too slow." At the end of the feedback period, the screen blanked and the participant was required to return to the start position without positional feedback from

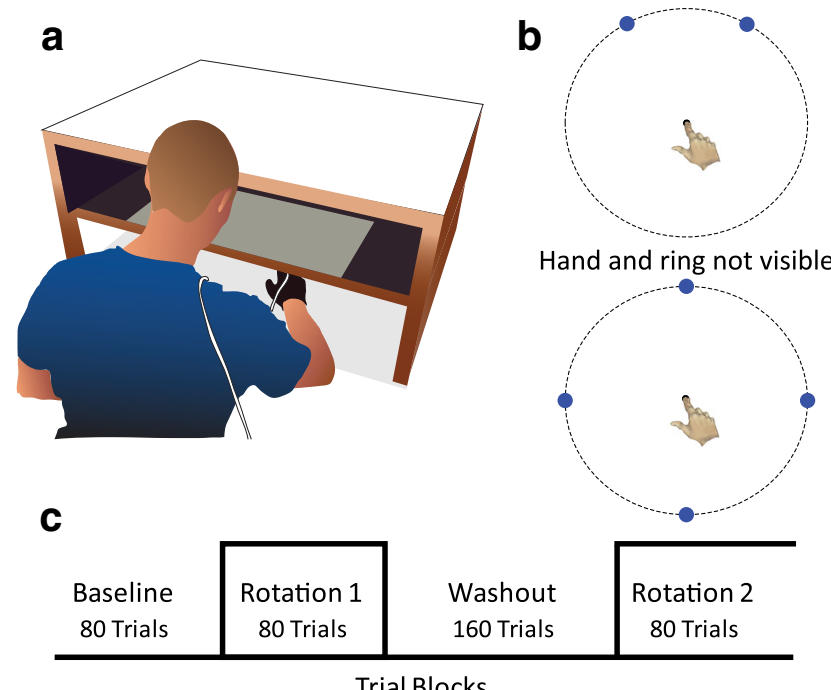

Trial Blocks

Figure 1. Experimental task. $\boldsymbol{a}$, Each participant slid his hand across a tabletop to hit visual targets. Vision of the hand was occluded by a mirror, which reflected a back-projected visual image to create the illusion that the hand and visual targets were coplanar. $\boldsymbol{b}$, Two- (top) and four- (bottom) target sets used in experiments. On each trial, one target was pseudorandomly selected. The participant reached from a central start location, attempting to "slice" through the target with the cursor. A trial was complete when the radial distance of the movement exceeded $10 \mathrm{~cm}$, indicated here by a dotted ring. $c$, Task design in Experiment 1 to assess savings. Veridical feedback was provided in the baseline and washout blocks. The visual feedback was perturbed during the two rotation blocks, with the size of the perturbation the same in each of these blocks.

the cursor. To guide the participant to the start position, a white ring with a radius equal to the distance of the hand from the start position was presented. This ring turned into the feedback cursor when the hand made contact with the central start position. The index finger had to remain within the start position for $200 \mathrm{~ms}$ before the presentation of the next target.

Experiment 1. Experiment 1 was designed to assess the effect of perturbation size on savings. Participants ( $n=10$ /group) were randomly assigned to one of four perturbation groups: $15^{\circ}, 30^{\circ}, 45^{\circ}$, or $60^{\circ}$. Within each group, 10 participants completed 400 trials, divided into four phases (Fig. 1c). The initial block of 80 trials served as a baseline to assess reaching performance in the absence of a perturbation. This was followed by an 80-trial initial adaptation block in which the cursor feedback was rotated in the counterclockwise direction (Rotation 1), with the size of the perturbation varied between groups. In the washout block, the rotation was turned off for 160 trials, allowing performance to return to baseline. The final block served as our probe of savings; here, the perturbation was reintroduced for another 80 trials (Rotation 2).

Experiment 2. Experiment 2 examined whether the savings observed with large perturbations results from faster adaptation of an internal model or from changes in action selection. For this experiment, we tested three groups ( $n=10 /$ group): one exposed to a $45^{\circ}$ rotation at the two target locations as Experiment 1, whereas the other two were exposed to either a $30^{\circ}$ or $45^{\circ}$ rotation at four targets spaced $90^{\circ}$ apart around $360^{\circ}$.

There were five blocks in this experiment. For the two-target group, the first four blocks were the same length and order as in Experiment 1, except that the Washout 1 block was only 100 trials long, and there was a 60 trial washout block after the Rotation 2 block. The perturbation size was fixed at $45^{\circ}$ for all participants in the Rotation 1 and Rotation 2 blocks. The fifth block was a second washout block (Washout 2), composed of 60 trials without a perturbation. In the four-target condition, the structure of the trial blocks was identical, except that the number of trials was adjusted in each block to accommodate the greater number of targets while keeping the total number of trials similar. The four-target tasks began with a Baseline of 40 trials, followed by Rotation 1 (100 trials), Washout 1 (120 trials), Rotation 2 (100 trials), and finally Wash- 
out 2 (40 trials). During the rotation blocks, participants were exposed to either a $30^{\circ}$ or $45^{\circ}$ visuomotor rotation. All other features of the fourtarget condition were identical to the two-target condition.

For all groups, we assessed savings over the initial trials of Rotation 2. To evaluate whether the participants were using an aiming strategy, the savings assessment was followed by a verbal intervention in the Rotation 2 block. After the seventh trial of this block in the two-trial condition, the program was paused. The experimenter instructed the participant that, "The perturbation that just turned on will now be off for the next two trials (see Fig. 3a). I want you to aim directly for the blue target and reach directly for the blue target on these trials." Following these two reaches, the participant was told that "the perturbation is back on" and the participant was allowed to finish the task without further interruption. For the four-trial conditions, the verbal intervention was introduced after trial 12 in the Rotation 2 block and participants performed a series of four trials, one to each target. The rotation was then reimposed for the remainder of the block.

Experiment 3. This experiment was designed to directly assay the contribution of an aiming strategy to savings in visuomotor adaptation tasks. To this end, we used the aim report technique of Taylor et al. (2014). Briefly, numbers were displayed along a ring (10 cm circumference) in $5.625^{\circ}$ increments, with the value increasing in the clockwise direction and decreasing in the counterclockwise direction, relative to the target position (see Fig. 4a). Beginning with the 21st trial in the baseline block, participants were required, at the beginning of each trial, to report their intended aim, verbally indicating the number closest to where they intended to reach. Aiming directly to the target was reported as "target" or " 0. " Participants were randomly assigned ( $n=10$ /group) to either a $15^{\circ}$ or $45^{\circ}$ rotation condition.

Each group completed five blocks of trials while reaching to four targets spaced in $90^{\circ}$ increments around $360^{\circ}$. The number of trials in each block was the same as the four-target conditions of Experiment 2: Baseline of 40 trials, followed by Rotation 1 (100 trials), Washout 1 (120 trials), and Rotation 2 (100 trials). At the end of Rotation 2, the program was paused and the numbers were removed from the screen. Participants were instructed to aim and reach directly to the visual location of the target for the remainder of the experiment (Washout 2, 40 trials).

Experiment 4. This experiment was designed to assess whether compensatory aiming could be controlled via an arbitrary cue, and whether the formation of such an action selection policy is dependent on the size of the perturbation. The Rotation blocks included both rotation and no-rotation trials. On trials in which the visual feedback was rotated, the cursor and return ring were presented in red. Interspersed within these rotation trials were no-rotation trials; here the cursor and return ring were white. Participants were told at the beginning of the session, "Later on, your cursor will occasionally turn red. This means something weird will happen on that trial but you are still trying to do the same thing, hit the target with your cursor."

Participants ( $n=10$ /group) were randomly assigned to either a $15^{\circ}$ or $45^{\circ}$ rotation group. Each group completed five blocks: Baseline, Rotation 1 , Washout 1 , Rotation 2 , and Washout 2 . The baseline was 80 trials long. The rotation blocks consisted of 70 rotation ("cued") trials in which the cursor was red and rotated and 10 ("uncued") trials in which the cursor was white and the rotation was turned off (see Fig. $5 a$ ). The 10 uncued trials were distributed unpredictably throughout the first rotation block. The same order of cued and uncued trials was repeated in the Rotation 2 block. The two rotation blocks were separated by 100 trials of washout. The final washout block was composed of 60 trials.

Experiment 5. In the final experiment, we examined how participants responded when presented with a novel perturbation, comparing conditions in which we expect an aiming strategy to be operative or not operative. Participants $(n=10$ /group) were randomly assigned to either a $15^{\circ}$ or $45^{\circ}$ rotation group, with the expectation that the $45^{\circ}$ group would use an aiming strategy. The trial structure was 80 baseline trials, 80 counterclockwise rotation trials (Rotation CCW), 160 trials of washout, and 80 trials of a $30^{\circ}$ clockwise rotation (Rotation CW) (see Fig. 6a). The second rotation is different in both direction and magnitude from the first rotation, providing a probe on "savings" in a novel context.
Data analysis. Movement duration was defined as the interval between when the finger exited the starting region and when the radial amplitude passed $10 \mathrm{~cm}$. We determined the reach trajectory between these two time samples. For each trial, position data was interpolated to 150 data points using a cubic spline. The Cartesian position data were converted to polar coordinates, and the radial component was numerically differentiated to determine movement speed independent of hand angle. Peak speed was calculated by smoothing the radial speed with a 75 -window loess function and taking the maximum value of this function. The hand angle (relative to target angle) at the position of peak speed was the primary dependent variable in all of the experiments. In Experiment 3 , we also performed analyses on the verbal aiming report data and the estimate of implicit adaptation.

Some of the experiments reported here used two targets spaced $60^{\circ}$ apart, while others used four targets, spaced in $90^{\circ}$ increments. Because of these differences, savings cannot be directly compared across these experiments. However, to standardize our savings metric, we adopted a common convention by creating a savings difference score using a measure that averaged over three reaches to each target: In two-target conditions, this was six trials; in the four-target conditions, this was 12 trials. The difference score for each condition was calculated by subtracting the mean heading angle for these three trials in the Rotation 1 block from the corresponding three trials in the Rotation 2 block. Before computing each difference score, the values were adjusted by subtracting the mean hand angle on the 10 null trials that preceded the associated rotation block. All $t$ tests are two-tailed unless otherwise noted. Statistical calculations were made with MATLAB 2009b (The MathWorks) ( $t$ tests) and SPSS 17 (ANOVAs).

In Experiments 1 and 5, we also determined the learning rate for each participant in the two rotation blocks by fitting an exponential function of the following form:

$$
a-b * e^{x * c}
$$

Parameter fitting was implemented with the built-in MATLAB function fminbnd to minimize squared error. The asymptotic performance parameter, $a$, was set to the mean of the last 5 trials. The parameter corresponding to the total amount of learning, $b$, was fixed as the difference between the mean of the last 5 trials and the first trial. The only free parameter was the learning rate constant, $c$ (Huang et al., 2011).

\section{Results}

\section{Experiment 1}

This experiment was designed to test whether savings varied as a function of the size of the perturbation, with different groups exposed to visuomotor rotations of $15^{\circ}, 30^{\circ}, 45^{\circ}$, or $60^{\circ}$. Participants in all four groups learned to compensate for the rotation by moving in a direction opposite of the visual perturbation (Fig. 2a). The visual error was substantially reduced by the end of the first rotation block (blue lines), although it fell short of full compensation.

As one measure of savings, we compared the rate of adaptation for the two rotation blocks (Fig. 2b). A mixed-factor ANOVA with the factors Rotation Size and Block revealed a within-subject effect of Block $\left(F_{(1,36)}=19.38, p<0.001\right)$ and a significant Block $\times$ Rotation Size interaction $\left(F_{(3,36)}=3.62, p=\right.$ 0.022 ). Bonferroni-corrected post hoc analyses revealed a faster learning rate in the second rotation for the $45^{\circ}$ and $60^{\circ}$ groups (one-tailed $t$ test, $t_{(9)}=4.3, p=0.001 ; t_{(9)}=3.1, p=0.007$ ), but not the $15^{\circ}$ and $30^{\circ}$ groups $\left(t_{(9)}=0.94, p=0.187 ; t_{(9)}=0.50, p=\right.$ $0.313)$. Thus, savings by this measure was limited to the two groups who experienced the largest perturbations. The same dissociation is observed if we use the difference score to estimate savings, limiting the analysis to six initial trials of the rotation blocks (Rotation 2 - Rotation 1 over these trials). Here, there was a significant effect of Rotation Size $\left(F_{(3,36)}=6.76, p=0.001\right)$, $\operatorname{Block}\left(F_{(1,36)}=25.2, p<0.001\right)$, and interaction of these factors, 

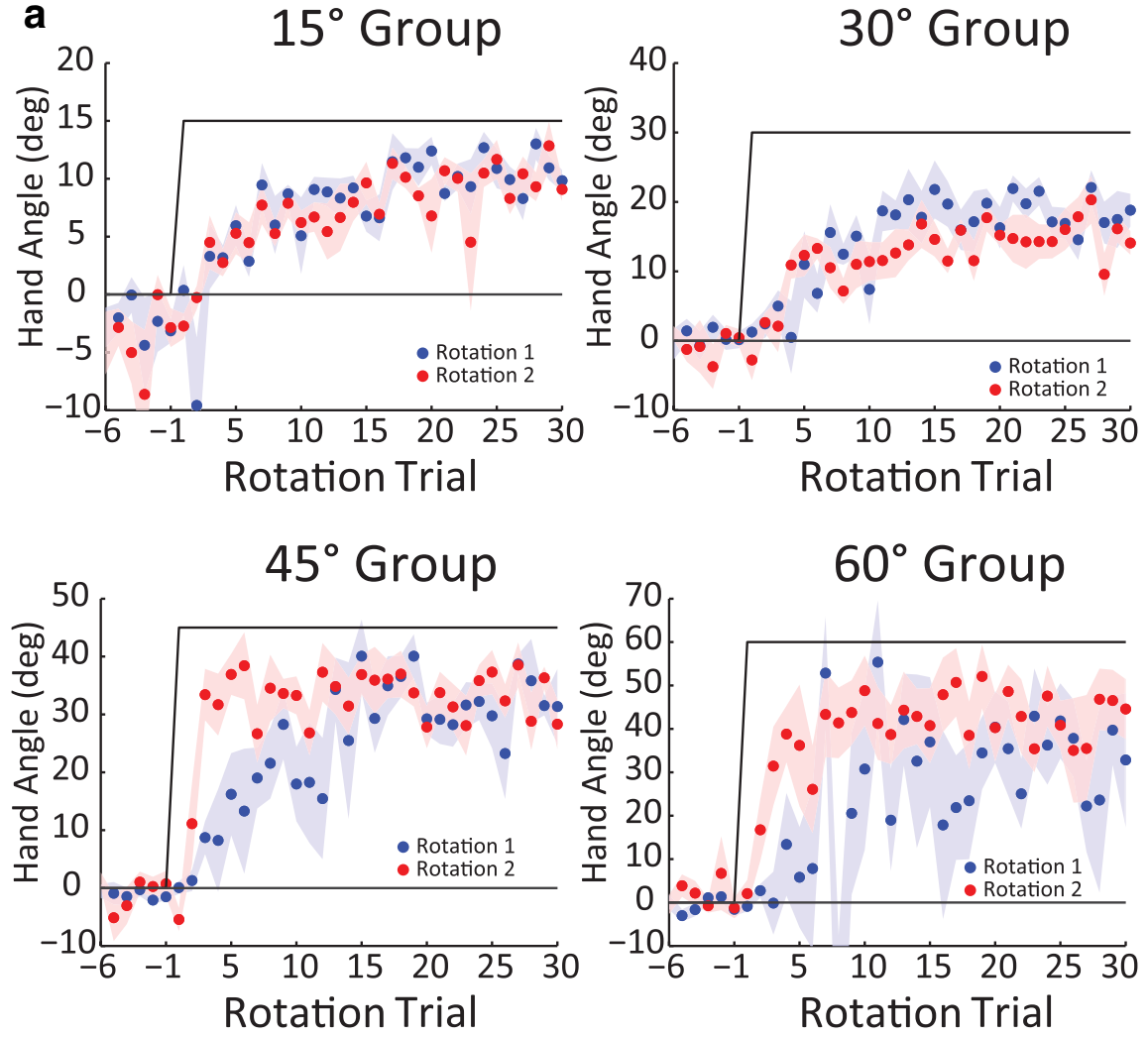

b

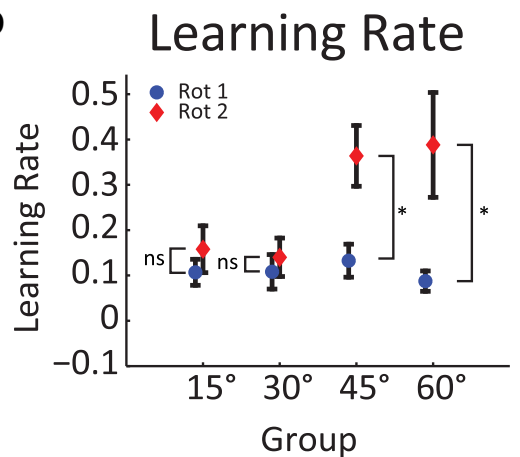

uted to an action selection process (Huang et al., 2011; Haith et al., 2015; Huberdeau et al., 2015; McDougle et al., 2015; Orban de Xivry and Lefévre, 2015). The current results are consistent with this view and indicate, more specifically, that a change in the selection of an aiming strategy causes savings. Indeed, our results raise the possibility that savings in sensorimotor adaptation tasks may be restricted to conditions that feature the prominent use of an aiming strategy.

\section{Experiment 2}

Experiment 2 was designed to indirectly assess the contribution of aiming to savings, building on the idea that aiming, an action selection process, can be under volitional control. To this end, the experiment was paused shortly into the second rotation block, and the participants were explicitly instructed to aim directly at the target. Our intention was to direct the participants to terminate the use of an aiming strategy if one was being used. In this manner, the initial trials of Rotation 2 allow us to assess savings, comparing performance here with that observed in the initial trials of Rotation 1 . The probe trials allowed us to assess the degree of true adaptation. If savings is due to adaptation, then the hand angle on these trials should remain similar to that seen in the initial trials of Rotation 2 (with a slight decay due to the pause required to give the instructions). In contrast, it would be parsimonious to attribute savings to re-aiming if participants exhibit a precipitous change in hand angle following the instructions.

In the two-target condition, participants showed faster relearning of the $45^{\circ}$ rotation (Fig. $3 b$ ). Over the first six trials of Rotation 2, the mean hand angle was $26.8^{\circ}$, a value that is $16.8^{\circ}$ larger than the value for the first six trials of Rotation 1 (paired $t$ test, $t_{(9)}=4.38, p=0.002$ ). When the participants were told that the perturbation was turned off and instructed to aim directly at the target,

Figure 2. Savings is only observed with large perturbations. $\boldsymbol{a}$, Heading angle of the hand relative to the target during last five baseline and first 20 trials of Rotation 1 (blue) and Rotation 2 (red) in Experiment 1. The data for the two rotation blocks are displayed in a superimposed manner for the four perturbation sizes. Note the different scaling of the vertical axis for each group. The $45^{\circ}$ and $60^{\circ}$ groups show faster relearning for the second rotation; this savings effect is absent for the $15^{\circ}$ and $30^{\circ}$ groups. $\boldsymbol{b}$, Mean learning rate from an exponential function independently fit to all trials in Rotation 1 and Rotation 2. c, Difference score measure of savings (Rotation 2 - Rotation 1). Data are mean \pm SEM. *Significant difference. ns, Not significant.

$\left(F_{(3,36)}=5.45, p=0.003\right)$. As can be seen in Figure $2 c$, savings was limited to the $45^{\circ}$ and $60^{\circ}$ groups.

The fact that savings was limited to the two groups presented with the largest perturbations is problematic for error-based models of visuomotor adaptation (with the exception of Berniker and Körding, 2011). These models predict that savings should be observed across different perturbation sizes; our results suggest a qualitative difference. Instead, we hypothesize that the differences in savings may arise from a process that is not adaptation, namely, the redeployment of an aiming strategy. It is increasingly recognized that participants may initially use strategies to compensate for salient perturbations in sensorimotor adaptation tasks (Redding and Wallace, 1993; Osu et al., 2002; Taylor et al., 2014). Building on this idea, it has recently been proposed that savings in these tasks can be attrib- there was a $30.4^{\circ}$ drop in heading angle between the two trials preceding the verbal intervention and the two intervention trials (Fig. 4b,c; paired $t$ test, $t_{(9)}=7.86, p<0.001$ ). The magnitude of this decline is considerably larger than what would be expected from temporal decay, which is only $20 \%$ of the adapted state (Hadjiosif and Smith, 2013). Rather, the flexible behavior observed here is consistent with the hypothesis that savings is related to a process under volitional control; participants recalled a previously successful aiming strategy to rapidly reduce target error without a large change in their sensorimotor mapping. The small, residual $4^{\circ}$ shift in hand angle observed during the probe trials, relative to the null trials preceding Rotation $2\left(95 \% \mathrm{CI}, 0.4^{\circ}, 7.5^{\circ}\right)$, likely reflects the 
degree of true adaptation that took place during the initial trials of Rotation 2.

To ensure that strategy use is not limited to tasks in which reaches are restricted to a limited part of the workspace, we repeated this experiment with four target locations spaced $90^{\circ}$ apart. Two groups were tested: one exposed to a $30^{\circ}$ rotation and the other exposed to a $45^{\circ}$ rotation (Fig. $3 \mathrm{c}$ ). The $45^{\circ}$ group showed reliable evidence of savings in the initial trials of Rotation 2, with a $17.4^{\circ}$ increase in the change in hand angle compared with the same trials in Rotation 1 (Fig. $4 d$; paired $t$ test, $t_{(9)}=$ 3.92, $p=0.004)$. In contrast, the $30^{\circ}$ group did not show reliable savings, with a modest $2.7^{\circ}$ change in hand angle over the first rotation (paired $t$ test, $\left.t_{(9)}=1.41, p=0.20\right)$. Furthermore, the two groups were different from one another (two-sample $t$ test, $t_{(18)}=3.06$, $p=0.007)$. Thus, consistent with Experiment 1 , savings was present for the $45^{\circ}$ group and absent or minimal for the $30^{\circ}$ group.

Both groups showed a significant decline in hand angle following the verbal intervention (Fig. $4 e ; 45^{\circ}$ group mean $=$ $20.7^{\circ}$, paired $t$ test, $t_{(9)}=3.94, p=0.003$; $30^{\circ}$ group mean $=8.3^{\circ}$, paired $t$ test, $t_{(9)}=$ $2.43, p=0.037)$. The decline was larger for the $45^{\circ}$ group compared with the $30^{\circ}$ group, although this difference was only marginally significant (two-sample $t$ test, $\left.t_{(18)}=1.96, p=0.066\right)$. Similar to the two-target condition, both groups showed small residual aftereffects (Fig. $4 c$; blue trials; $45^{\circ}$ group mean $=2.7^{\circ}$, paired $t$ test, $t_{(9)}=2.59, p=0.029 ; 30^{\circ}$ group mean $\left.=3.4^{\circ}, t_{(9)}=4.02, p=0.003\right)$. The small aftereffects indicate that, not only can participants flexibly deploy an aiming strategy, but the performance gains from aiming dwarf those of adaptation.

The results from the verbal intervention in the $30^{\circ}$ group are puzzling: Although these participants showed a drop in hand angle, indicative of aiming, they did not show reliable savings. Previous studies have reported savings with this size perturbation (Huang et al., 2011; Hadjiosif and Smith, 2013; Herzfeld et al., 2014; Haith et al., 2015; Huberdeau et al., 2015; McDougle et al., 2015; Orban de Xivry and Lefévre, 2015). In the current results, the participants did exhibit savings, apparently because they aimed the same amount in the early phase of both rotations. In contrast, the $45^{\circ}$ groups showed a larger change in aim from Rotation 1 to Rotation 2. We return to this a

\begin{tabular}{|c|c|c|c|c|c|}
\hline $\begin{array}{l}\text { Baseline } \\
80 \text { Moves }\end{array}$ & $\begin{array}{l}\text { Rotation } \\
80 \text { Moves }\end{array}$ & $\begin{array}{l}\text { Washout } \\
100 \text { Moves }\end{array}$ & $\begin{array}{l}\text { Rotation } \\
80 \text { Mov. }\end{array}$ & $\begin{array}{l}\text { Washout } \\
60 \text { Moves }\end{array}$ & Two Target \\
\hline (40) & $(100)$ & $(120)$ & (100) & $(40)$ & (Four Target) \\
\hline
\end{tabular}

b Two Target $45^{\circ}$ Group

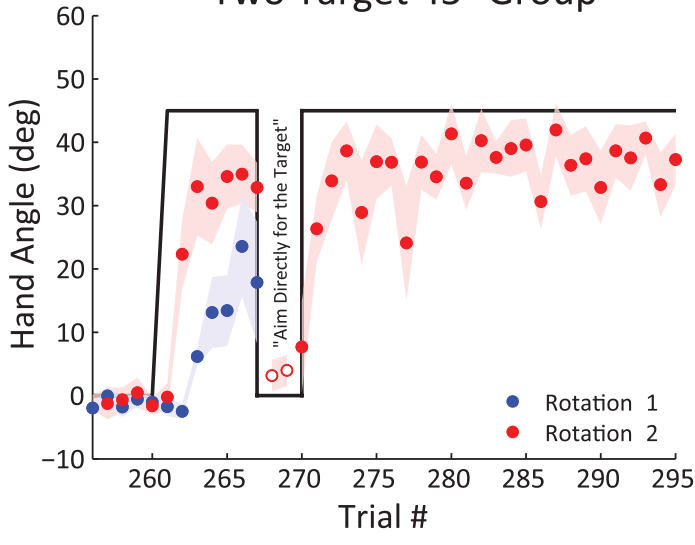

Two Target

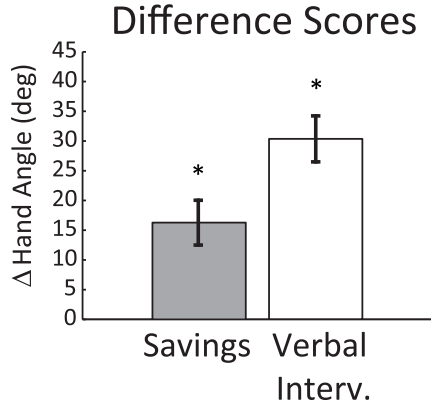

C Four Target $30^{\circ}$ Group
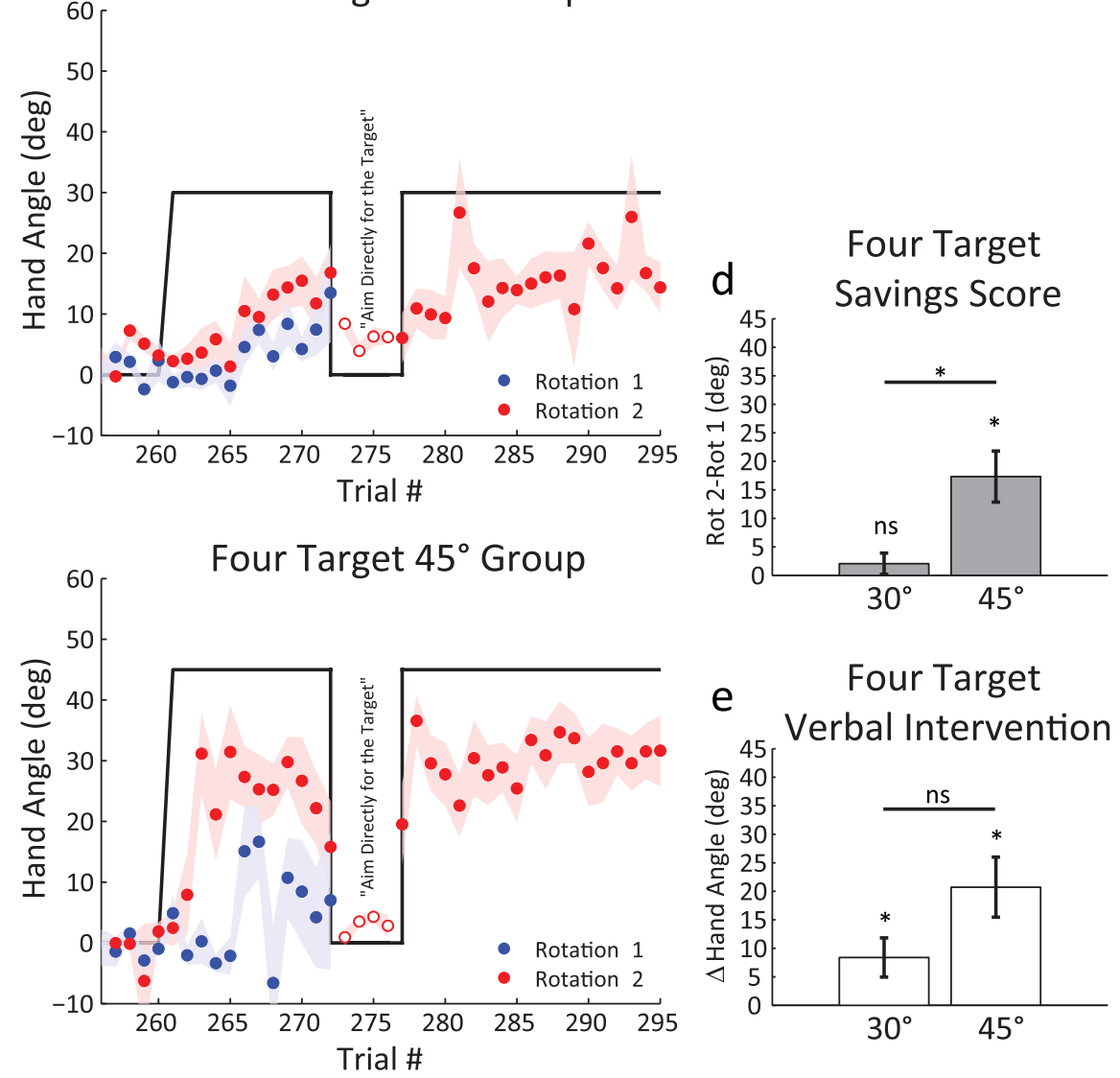

Figure 3. Gains from savings are abolished by instructions to aim directly for the target. $a$, Trial schematic for Experiment 2 , denoting the verbal intervention following the seventh trial of the Rotation 2 block. For the following 2 trials (blue bar), participants were instructed to aim and reach directly for the target. $\boldsymbol{b}$, Left, Behavior during the Rotation 2 block for the two-target $45^{\circ}$ group. There is an initial period of rapid relearning, followed by a sharp change in hand angle in response to the verbal intervention. Right, Difference score between the first 6 trials of Rotation 1 and Rotation 2 blocks (red) and between the average heading angle for the 2 intervention trials and the 2 trials preceding the intervention (blue). $c$, Time course of hand angles for the $45^{\circ}$ (top) and $30^{\circ}$ (bottom) groups in the four-target groups. Savings score $(\boldsymbol{d})$, defined by the difference between the first 12 trials of the Rotation 1 and Rotation 2 blocks and (e), verbal intervention scores (using the 4 intervention trials and 4 preintervention trials). While savings was only reliable for the $45^{\circ}$ group, there was a drop in hand angle following the verbal intervention for both groups. Data are mean \pm SEM. * Significant difference. ns, Not significant. 
a

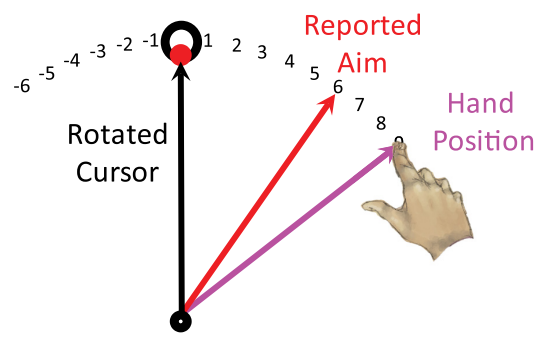

b

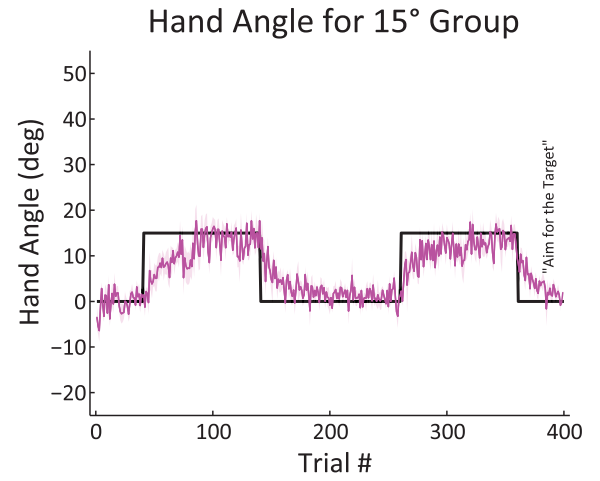

C

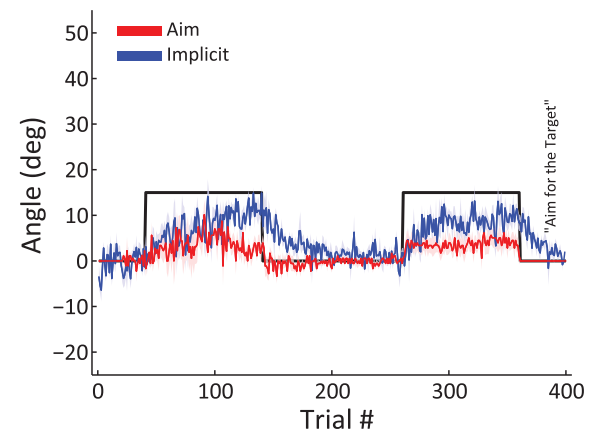

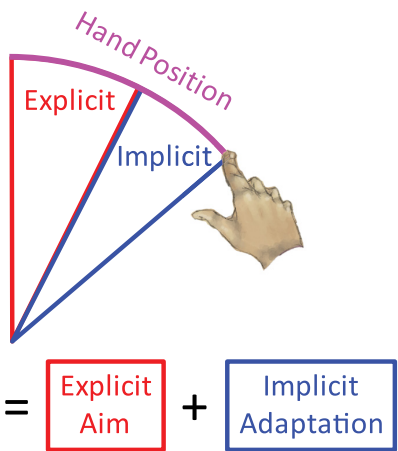

Hand Angle for $45^{\circ}$ Group

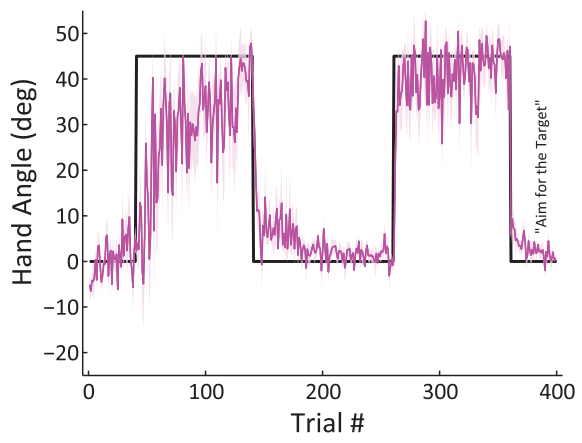

Explicit/Implicit for $45^{\circ}$ Group

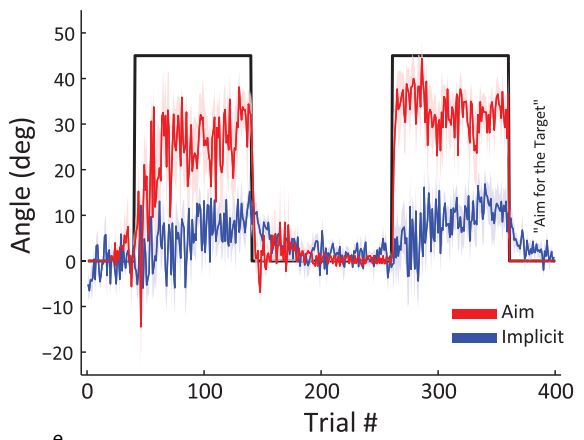

e Savings over all Measures

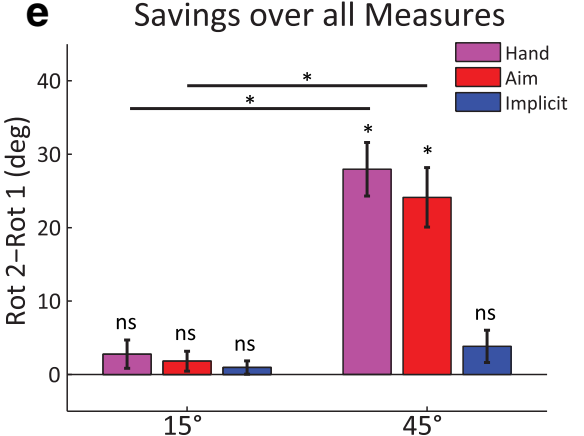

Figure 4. Savings assessed with aiming report task in Experiment 3. $\boldsymbol{a}$, Left, Task schematic. Numbers were visible in ascending and descending manner around the target. Participant verbally reported the number corresponding to his/her aiming location and then performed the reach. Right, Assuming performance (heading angle) is the composite of an explicit aiming strategy and implicit adaptation, the magnitude of the latter can be estimated via subtraction. $\boldsymbol{b}$, Overall behavior for $15^{\circ}$ (left) and $45^{\circ}$ (right) groups. c, Mean aiming reports (red) and implicit adaptation (blue, estimated via subtraction). Participants were instructed to aim directly for the target in the final washout block. $\boldsymbol{d}$, Aiming reports over the first 30 trials, superimposed for Rotation 1 and Rotation 2. e, Savings difference scores for each measure. Data are mean \pm SEM. * Significant difference. ns, Not significant.

issue in the Discussion. Taken together, the results of Experiment 2 indicate that the savings observed early in a second rotation can be attributed to aiming behavior that is under volitional control. The small residual aftereffects observed during the intervention trials suggest that aiming and adaptation occur in parallel.

\section{Experiment 3}

This experiment directly measured aiming behavior in the context of savings, asking participants to verbally report their aiming location before each reach (Taylor et al., 2014). Participants were randomly assigned to either a $15^{\circ}$ or $45^{\circ}$ group to assess changes 
in aim across different magnitudes of visuomotor rotation. The trial design was similar to the four-target conditions of Experiment 2, with two 100 trial rotation blocks separated by a 120 trial washout. Aiming reports were initiated halfway through the baseline block and were assessed on every trial until the end of the second rotation. In a final washout block, the numbers were removed from the screen and participants were instructed to move their hands directly to the target (Taylor et al., 2014).

We excluded trials where the participant reported an aiming location that was more than 2 times the perturbation size, as well as trials in which the discrepancy between the reported aiming location and actual hand position was $>45^{\circ}$. On average, this excluded $3.0 \%$ of trials in the $45^{\circ}$ group and $2.2 \%$ of trials in the $15^{\circ}$ group. Most of these trials came from three individuals ( 1 in the $45^{\circ}$ group, 2 in the $15^{\circ}$ group), but even for these individuals, the percentage did not exceed $10 \%$ of their total aiming trials. Statistical tests of savings were not meaningfully affected by the exclusion of these trials.

As in Experiment 1, the hand angle difference score indicated reliable savings for the $45^{\circ}$ group (Fig. $4 b$,e; mean $=28^{\circ}$, paired $t$ test, $t_{(9)}=7.67, p<0.001$ ), but not for the $15^{\circ}$ group (mean $=2.7^{\circ}$, paired $t$ test, $t_{(9)}=1.43, p=0.19$ ). This savings pattern was mirrored in the aiming report data: Participants in the $45^{\circ}$ group showed a reliable increase in the reported aiming location for Rotation 2 compared with Rotation 1 (mean $=24^{\circ}$, paired $t$ test, $t_{(9)}=5.94, p<$ 0.001). Participants in the $15^{\circ}$ group did not show a consistent change in the reported aiming location between the two rotation blocks $\left(\right.$ mean $=1.8^{\circ}$, paired $t$ test, $t_{(9)}=1.33, p=0.21$ ).

To estimate the amount of implicit adaptation, we subtracted each participant's reported aim from the hand angle. We then calculated savings using the difference score measure with these data. Interestingly, neither group showed savings in the estimate of implicit adaptation ( $45^{\circ}$ group, paired $t$ test, $t_{(9)}=1.74, p=$ $0.115 ; 15^{\circ}$ group, paired $t$ test, $\left.t_{(9)}=1.06, p=0.31\right)$. Moreover, for both the overall performance and aiming report measures, savings in the $45^{\circ}$ group was different from the $15^{\circ}$ group (reaching: two-sample $t$ test, $t_{(18)}=6.1, p<0.001$; aiming: two-sample $t$ test, $\left.t_{(18)}=5.2, p<0.001\right)$, but not in the measure of implicit adaptation (two-sample $t$ test, $t_{(18)}=1.2, p=0.242$ ). All $t$ tests here were Bonferroni corrected.

The results of Experiment 3 provide direct evidence that savings in visuomotor adaptation is associated with how participants change their aiming strategy when re-encountering a perturbation, and not associated with faster modification or recall of an internal model. Additionally, the results indicate that the larger performance changes observed with large perturbations is primarily due to changes in the explicit aiming process. Indeed, the magnitude of implicit adaptation appears to be relatively constant for the small and large perturbations (Bond and Taylor, 2015).

\section{Experiment 4}

Prior studies have shown that, without extensive training, processes involved in sensorimotor adaptation are insensitive to arbitrary static cues (Cunningham and Welch, 1994; Miall et al., 2004; Howard et al., 2013). For example, people have great difficulty simultaneously learning internal models for clockwise and counterclockwise rotations when the context is cued by color; under such conditions, there is substantial interference between the two contexts. However, with minimal training, people are extremely good at using novel cues to select arbitrary actions.

We exploited this difference between action selection and adaptation by designing an experiment where the presence of a visuomotor rotation was indicated by an arbitrary color cue.
These perturbation trials were interspersed with trials in which the rotation was absent (Fig. $5 a$ ). We reasoned that if participants were employing an aiming strategy, they should be able to use the color cue to determine whether or not to use the strategy. In contrast, the color cue should have minimal effect on behavioral changes caused by adaptation.

We again compared two groups: one exposed to a $15^{\circ}$ rotation and the other exposed to a $45^{\circ}$ rotation. Based on the results of Experiment 1, we assume that the $45^{\circ}$ group would use a combination of error-based adaptation and aiming. As such, their performance should switch rapidly between the cued and uncued trials during the rotations and, more important, that the color cue will result in savings on the second rotation block. Indeed, we would expect this savings to be evident on the first trial of the second rotation block, even before the perturbation has been re-experienced. In contrast, we expected to observe little flexibility in the performance of the $15^{\circ}$ group.

Figure $4 b$ shows the time series for the two groups. There is a clear separation between hand angle on the cued and uncued trials for the $45^{\circ}$ group, whereas there is considerable overlap for the $15^{\circ}$ group. We quantified the effect of the cue by creating trial pairs, with each pair composed an uncued trial from the rotation blocks and its immediately preceding cued trial. These data were evaluated in a Rotation Size $\times$ Block $\times$ Trial Type (Cued/Uncued) mixed ANOVA (Fig. $5 d$ ). The main effects of Rotation Size, $F_{(1,18)}=46.64, p=<0.001$, and Block, $F_{(1,18)}=48.88, p<0.001$, were reliable, whereas the effect of Trial Type was not, $F_{(1,18)}=$ $0.73, p=0.405$. A three-way Block $\times$ Rotation Size $\times$ Trial Type interaction was reliable, $F_{(1,18)}=6.54, p=0.02$, driven by simple effects of Block and Trial Type in the $45^{\circ}$ group $\left(F_{(1,9)}=76.07\right.$; $\left.p<0.001, F_{(1,9)}=5.78, p=0.03\right)$ but not the $15^{\circ}$ group $\left(F_{(1,9)}=\right.$ $\left.1.36, p=0.259 ; F_{(1,9)}=1.43, p=0.247\right)$. These results indicate a difference between the cued and uncued trials for the $45^{\circ}$ group, but not the $15^{\circ}$ group, as well as an improvement in performance on cued trials in the second rotation for the $45^{\circ}$ group.

Performance on the uncued trials for both groups shows a gradual increase in hand angle within a rotation block. Moreover, the magnitude of this effect is similar across both rotation blocks and across the two groups. Coupled with the results of Experiment 3 , this pattern is consistent with the ongoing operation of adaptation, operating with relative indifference to the size of the perturbation. We assume that this adaptation occurs even on cued trials because there remains a substantial error between the strategic aiming location and the position of cursor feedback (Mazzoni and Krakauer, 2006). The current design provides a demonstration of the parallel operation of strategic and adaptation processes (Taylor and Ivry, 2011) in a task setting that does not overtly instruct participants to aim somewhere other than the target.

We assume that the cued trials are composed of both reaiming and adaptation, whereas the uncued trials reflect adaptation alone. If aiming strategies are being flexibly invoked by this arbitrary cue, we should be able to trigger savings with the cue alone. To test this prediction, we focused on the first trial of the second rotation. This trial offers a powerful test of the re-aiming hypothesis because the participants have yet to experience the error associated with the reintroduction of the visuomotor rotation. Therefore, if savings is observed on this trial, it can only represent a change in the selected action and not error-based adaptation.

To assess this cue-evoked savings, we computed a difference score using only the first trial of Rotation 1 and Rotation 2 (Fig. $5 e$ ). The $15^{\circ}$ group did not show evidence of cue-evoked savings 

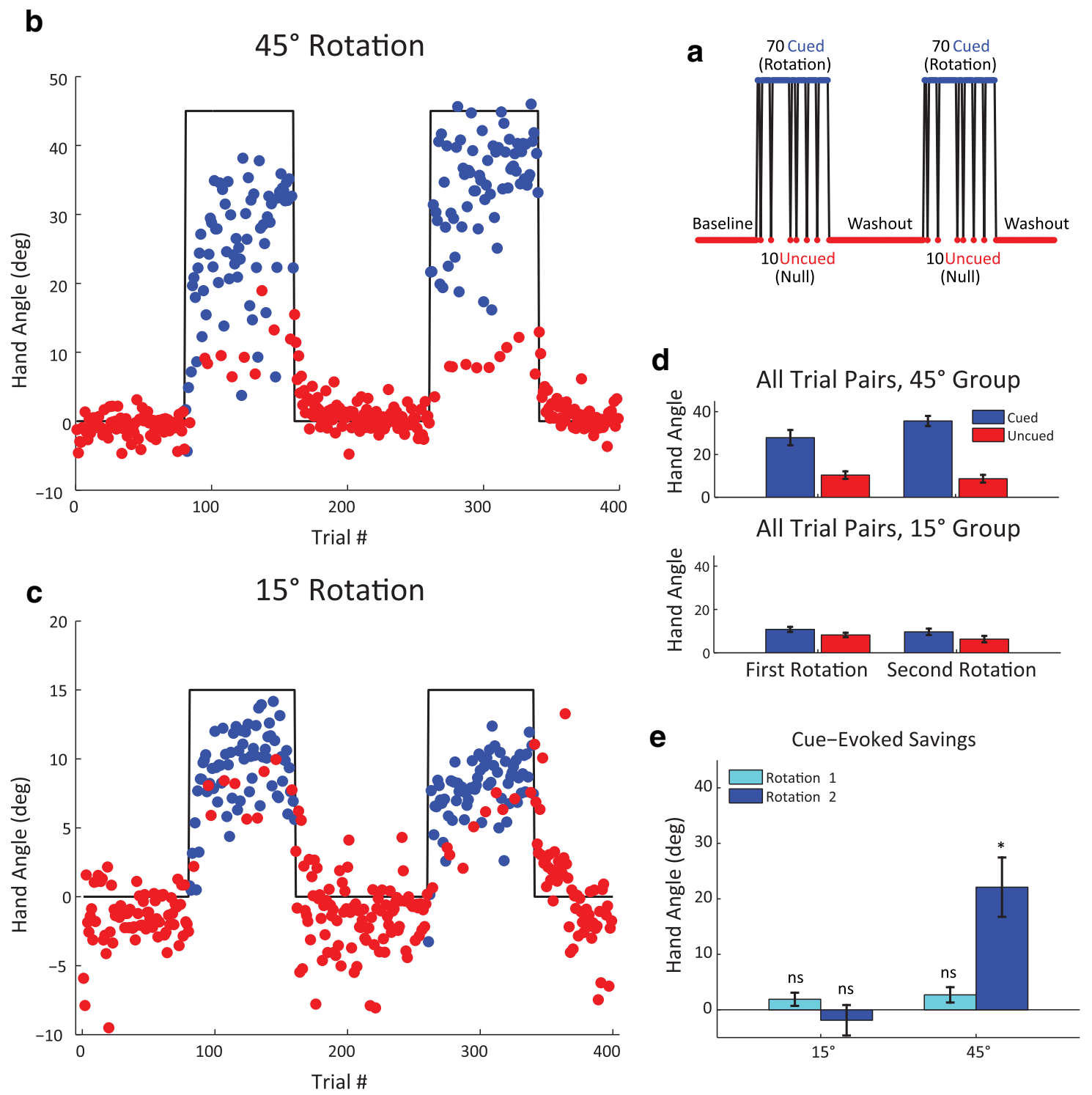

Figure 5. Control of aiming strategy by an arbitrary color cue. $\boldsymbol{a}$, Interleaved design of Experiment 4 in which the cursor changed color on rotation trials (depicted in blue). The cursor remained white on no-rotation trials (depicted in red). $\boldsymbol{b}, \boldsymbol{c}$, Heading angle for groups exposed to $45^{\circ}$ and $15^{\circ}$ rotations, respectively. Note the difference in the scale of the $y$-axis. $\boldsymbol{d}$, Blue bars represent mean of the cued trials, limited to those that immediately preceded an uncued trial. Red bars represent mean of uncued trials.e, Baseline subtracted hand angle on the first trial for Rotation 1 and Rotation 2. The color cue prompted the use of an aiming strategy for the $45^{\circ}$ group, even before the participants had experienced errors caused by the perturbation in Rotation 2 . Data are mean \pm SEM . *Significant difference. ns, Not significant.

$\left(\right.$ mean $=-3.8^{\circ}$, paired $t$ test, $\left.t_{(9)}=1.41, p=0.19\right)$. In contrast, the $45^{\circ}$ group displayed significant cue-evoked savings ( mean $=$ $19.4^{\circ}$, paired $t$ test, $\left.t_{(9)}=3.51, p=0.007\right)$. Furthermore, the two groups were different from each other (two-sample $t$ test using Satterthwaite's approximation for unequal variances, $t_{(18)}=$ $3.77, p=0.002)$. This cue-evoked savings in the $45^{\circ}$ group was not the result of online feedback corrections: Individual reach trajectories were straight (data not shown), hand position was measured at peak radial speed, and the average movement time was $208 \mathrm{~ms}$, a value unchanged from the mean movement time of the 10 null trials previous to Rotation 2 onset (paired $t$ test, $t_{(9)}=$ $0.26, p=0.80$. In summary, this "first trial" analysis provides a powerful demonstration that savings in visuomotor adaptation reflects the use of an aiming strategy and that this process is not contingent on experiencing previously encountered errors.

\section{Experiment 5}

In the final experiment, we examined how an aiming plan generalizes to a novel perturbation. We exposed participants to either a $15^{\circ}$ or $45^{\circ}$ counterclockwise rotation in the first block and then tested both groups with a clockwise $30^{\circ}$ rotation in the second rotation block (Fig. $6 a$ ). Given the absence of savings for a $15^{\circ}$ rotation observed in Experiments 1, 3, and 4, we expect that this group will provide a baseline measure of learning a reversed $30^{\circ}$ rotation. The key question is what happens when the $45^{\circ}$ group is tested with the reversed rotation. It may be that, given the novel context, there will be little benefit from the initial experience with a rotation; as such, performance will be similar to that exhibited by the participants who had been exposed to an initial $15^{\circ}$ rotation. Alternatively, having developed a strategy to compensate for the initial $45^{\circ}$ rotation, these participants may rapidly invoke a strategy when encountering the new perturbation. 


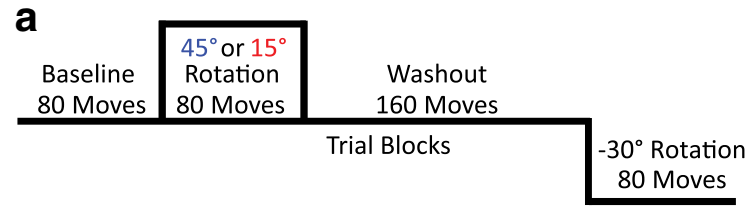

b
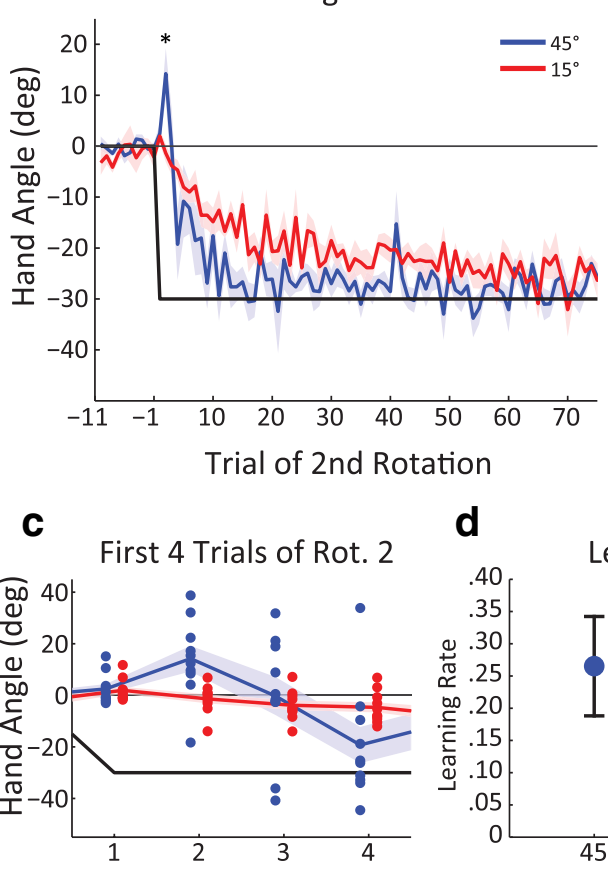

Trial of 2nd Rotation

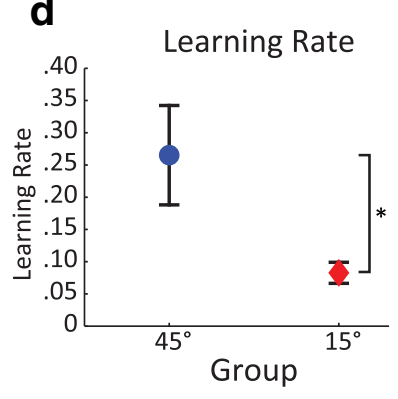

Figure 6. Inappropriate recall of aiming strategies in response to a novel perturbation. $\boldsymbol{a}$, In Experiment 5 , all participants were exposed to a novel $-30^{\circ}$ rotation after completing a training block with either $a 5^{\circ}$ or $15^{\circ}$ rotation, followed by a washout block. $\boldsymbol{b}$, Heading angle for the last 10 trials of the washout block and all trials of the Rotation 2 block. The $45^{\circ}$ group initially responded to the perturbation by moving in the wrong direction and, after a few trials, reversed direction, reducing error faster than the $15^{\circ}$ group. $c$, Enhanced picture of behavior over the first four trials of the Rotation 2 block, showing performance of each individual (dots). Note the expanded vertical axis relative to $\boldsymbol{b}$. $\boldsymbol{d}$, Mean learning rate from an exponential fit of all trials in Rotation 2 block, showing faster learning of the rotation for the $45^{\circ}$ group. Data are mean \pm SEM. *Significant difference.

Both groups showed good learning in the first rotation block and returned fully to baseline before the second rotation (Fig. $6 b$; trials -11 to -1 ). Their performance in the Rotation 2 block was quite different. The group that had initially been exposed to the $15^{\circ}$ rotation showed a standard adaptation function, with visual error decreasing in a monotonic fashion. In contrast, there were two striking features in the performance of the group who had been exposed to the $45^{\circ}$ rotation in the Rotation 1 block. First, on the second trial of Rotation 2, 9 of 10 participants moved in a direction that amplified, rather than reduced, the visual error (Fig. $6 c, d$; one-sample $t$ test, $\left.t_{(9)}=2.87, p=0.02\right)$. This behavior is the opposite of what would be expected from an error-based adaptation process. Rather, when faced with the new perturbation, these participants appeared to redeploy an aiming strategy that had been useful in the previous context. Second, the participants quickly abandoned this strategy and moved in a direction that rapidly compensated for the $30^{\circ}$ rotation. Indeed, relative to the $15^{\circ}$ group, these participants subsequently showed faster learning of the $30^{\circ}$ rotation.
We quantified learning rate by fitting a single exponential function over all trials of the Rotation 2 block. The learning rate parameter for the $45^{\circ}$ group was larger than the learning rate of the $15^{\circ}$ group (two-sample $t$ test, $t_{(18)}=2.97, p=0.04$; Fig. $5 e$ ), indicating a faster change in behavior over the same number of trials. We propose that the participants who were initially trained with a $45^{\circ}$ rotation were sensitized to invoke and adjust an aiming strategy upon encountering the novel rotation.

\section{Discussion}

Recent work shows that the behavior in sensorimotor adaptation tasks arises from both error-based adaptation and other learning processes and that these other processes may be responsible for savings (Huang et al., 2011; Herzfeld et al., 2014; Taylor et al., 2014; Haith et al., 2015; Huberdeau et al., 2015; McDougle et al., 2015). Here we examined the contribution of aiming strategies to savings upon relearning in visuomotor adaptation tasks. Our results converge on two key ideas. First, savings reflects recall in the action selection domain, with participants invoking an aiming strategy when a perturbation was reintroduced. Second, errorbased adaptation does not appear to make any contribution to savings in visuomotor adaptation. This dissociation was observed whether we used indirect or direct methods to partition behavior into strategic and adaptation components.

\section{Savings as action selection}

Our results are best described by the hypothesis that savings in visuomotor tasks arises from the recall of an aiming strategy. In a sense, the term "savings" here is a misnomer, given the classic definition of faster relearning after forgetting (Ebbinghaus, 1913). We assume that the participants never forgot the strategy but determined when or when not to use the strategy. Experiments 4 and 5 shed light on factors that can evoke aiming upon re-encountering a rotation. In both experiments, the strategy was recalled without re-exposure to the errors that drove its development in the first rotation. In Experiment 4, the aiming strategy was evoked by a color cue; in Experiment 5, it was an error of opposite sign to the previously experienced error. This suggests that, in typical savings designs, such as Experiment 1 (A-Null-A), the large error can become a cue for the recall of aiming strategies (Huberdeau et al., 2015). The Experiment 3 results are also consistent with this idea, as re-aiming in the $45^{\circ}$ group occurred immediately after a single perturbation trial (Fig. $4 d$ ).

Huang et al. (2011) have also proposed an action selection account of savings, arguing that savings arises from the memory of hand positions associated with successful performance during earlier presentations of a perturbation. This conceptual model would offer a different interpretation of some of our results. For example, in Experiment 5, the $45^{\circ}$ group produced an initial shift in movement direction that had been successful in the first rotation block. We attribute this to inappropriate recall of an aiming strategy rather than a memory for hand position, but we cannot discriminate between these hypotheses with our results. Other results from the current study, however, do not align with predictions derived from the Huang model. First, their model predicts savings for all perturbation magnitudes and fails to predict the changes in aim observed in Experiment 3. Moreover, the $45^{\circ}$ group in Experiment 5 showed faster learning of a novel rotation with a hand space solution that had never been experienced by the participants. More recently, Haith et al. (2015) have shown that savings is only observed when participants are given adequate time to prepare a movement and that savings can be observed with a small number of initial learning trials (Huberdeau 
et al., 2015). These findings are consistent with our conclusion that aiming strategies are responsible for savings.

\section{The role of perturbation size}

We only observed savings with large perturbations. It is tempting to conclude that this is because aiming does not occur when the perturbation is small. However, the aiming report data for the $15^{\circ}$ group in Experiment 3 revealed small, yet consistent, deviations from the target location. Bond and Taylor (2015) have also shown that participants use aiming strategies across a large range of rotations. Given that some form of aiming occurs with small rotations, we surmise that use of an aiming strategy alone is not responsible for savings. Rather, savings may depend on a substantial change in aiming strategy between the beginning and end of the first rotation, the absolute angular magnitude of the aiming strategy, or the relative proportion of aiming and implicit adaptation.

Unlike the results from the current experiments, other studies have reported savings with $30^{\circ}$ rotations (Caithness et al., 2004; Krakauer et al., 2005; Huang et al., 2011; Hadjiosif and Smith, 2013; Herzfeld et al., 2014; Haith et al., 2015; Huberdeau et al., 2015; Orban de Xivry and Lefévre, 2015). We are unable to offer a straightforward explanation for this discrepancy. There can be differences between the experimental setups of different laboratories as well as differences in the instructions. These factors can affect how participants understand the task, which may in turn affect how they respond to a perturbation. Our data suggest that participants in our setup actively use aiming strategies in response to $30^{\circ}$ rotations (see also Bond and Taylor, 2015), but their strategy was of similar size for both Rotations 1 and 2. Importantly, our central claim, that savings is associated with action selection rather than error-based adaptation, is not dependent on the idea that there is an absolute point at which aiming or savings becomes operative.

\section{Relationship to error-based models of savings}

Our account of savings in visuomotor adaptation tasks stands in contrast to many error-based models of savings. Smith et al. (2006) presented a model in which error-based adaptation operates at two time scales. Savings comes about as a result of the interaction of these two processes. This model makes clear predictions that the degree of savings will be independent of rotation size and only observed when the slower process has not returned to a baseline state. Counter to these predictions, we observed savings in groups exposed to large rotations after a long washout where the slow process should have returned to baseline (based on parameters from Zarahn et al., 2008). Berniker and Körding (2011) also proposed a two-process model, one in which small and large errors are treated differently. Critically, the Berniker model cannot account for savings observed when an aiming strategy is evoked by a cue rather than re-exposure to an error. As such, although we also argue that savings reveals the operation of multiple processes, we favor a model entailing the operation of two qualitatively distinct processes: an error-based adaptation process to ensure accurate response execution and an aiming process to optimize response selection.

Herzfeld et al. (2014) recently published an alternative errorbased model of savings. The core idea is that errors are used to adapt an internal model of the correct movement, but also use error information to modulate future learning. Savings comes about because the system is sensitized to familiar errors. This model fails to predict a number of the results from the current study. First, the model cannot account for our finding that savings depends on rotation magnitude. Second, the model does not account for the volitional nature of aiming and its impact on savings. Third, unlike the behavior in Experiment 4, the model requires re-experiencing errors to exhibit savings. Finally, although the model does offer a novel account of generalization in savings, it would not produce an inappropriate response to a novel rotation as observed in Experiment 5.

\section{Awareness and aiming}

We have intentionally skirted the role of awareness with respect to the contrast of aiming and adaptation. In our experiments, perhaps most salient in Experiment 2 and 3, aiming is under volitional control. Indeed, we assume that the processes involved in the use of aiming strategies are generally accessible to awareness even when not directly probed, whereas adaptation always operates in an implicit manner. Although aiming strategies and adaptation differ in terms of their accessibility to awareness, we do not believe this is the defining distinction between them, choosing to focus instead on their mapping to action selection and movement execution, respectively. We expect there are features of action selection that are not, or need not be, explicit. For example, action selection may be modified through operant mechanisms in the absence of awareness (Pessiglione et al., 2008). Similarly, it is likely that most participants were aware that the environment was perturbed following the introduction of $15^{\circ}$ or $30^{\circ}$ rotations in our experiments, but this did not lead to the rapid reinstantiation of an aiming strategy when the perturbation was reintroduced. Therefore, we do not consider awareness to be the defining distinction between adaptation and action selection in the context of savings.

In conclusion, it is becoming clear that motor learning results from a panoply of learning processes that allow flexible behavior in varied environments (Wolpert et al., 2011; Taylor et al., 2014; Huberdeau et al., 2015). The current results highlight that within-session savings for a visuomotor adaptation task is accounted for by action selection (Huang et al., 2011; Haith et al., 2015; Huberdeau et al., 2015; McDougle et al., 2015; Orban de Xivry and Lefévre, 2015), arising from the recall of an aiming strategy. As striking, we failed to observe any savings in conditions in which learning appeared to be restricted to error-based adaptation.

\section{References}

Berniker M, Körding KP (2011) Estimating the relevance of world disturbances to explain savings, interference and long-term motor adaptation effects. PLoS Comput Biol 7:e1002210. CrossRef Medline

Bond KM, Taylor JA (2015) Flexible explicit but rigid implicit learning in a visuomotor adaptation task. J Neurophysiol 113:3836-3849. CrossRef Medline

Caithness G, Osu R, Bays P, Chase H, Klassen J, Kawato M, Wolpert DM, Flanagan JR (2004) Failure to consolidate the consolidation theory of learning for sensorimotor adaptation tasks. J Neurosci 24:8662-8671. CrossRef Medline

Cunningham HA, Welch RB (1994) Multiple concurrent visual-motor mappings: implications for models of adaptation. J Exp Psychol 20:987. CrossRef Medline

Ebbinghaus H (1885/1913) Memory: contribution to experimental psychology (No. 3). New York: Teachers College, Columbia University.

Haith AM, Huberdeau DM, Krakauer JW (2015) The influence of movement preparation time on the expression of visuomotor learning and savings. J Neurosci 35:5109-5117. CrossRef Medline

Hadjiosif A, Smith MA (2013) Savings is restricted to the temporally labile component of motor adaptation. Proc Transl Compute Mot Control (San Diego, CA), 2013.

Haruno M, Wolpert D, Kawato M (2001) Mosaic model for sensorimotor learning and control. Neural Comp 13:2201-2220. CrossRef Medline

Herzfeld DJ, Vaswani PA, Marko MK, Shadmehr R (2014) A memory of 
errors in sensorimotor learning. Science 345:1349-1353. CrossRef Medline

Howard IS, Wolpert DM, Franklin DW (2013) The effect of contextual cues on the encoding of motor memories. J Neurophysiol 109:2632-2644. CrossRef Medline

Huang VS, Haith A, Mazzoni P, Krakauer JW (2011) Rethinking motor learning and savings in adaptation paradigms: model-free memory for successful actions combines with internal models. Neuron 70:787-801. CrossRef Medline

Huberdeau DM, Haith AM, Krakauer JW (2015) Formation of a long-term memory for visuomotor adaptation following only a few trials of practice. J Neurophysiol 114:969-977. CrossRef Medline

Krakauer, J W (2009) Motor learning and consolidation: the case of visuomotor rotation. In: Progress in motor control, pp 405-421. New York: Springer.

Krakauer JW, Ghez C, Ghilardi MF (2005) Adaptation to visuomotor transformations: consolidation, interference, and forgetting. J Neurosci 25: 473-478. CrossRef Medline

Lee JY, Schweighofer N (2009) Dual adaptation supports a parallel architecture of motor memory. J Neurosci 29:10396-10404. CrossRef Medline

Mazzoni P, Krakauer JW (2006) An implicit plan overrides an explicit strategy during visuomotor adaptation. J Neurosci 26:3642-3645. CrossRef Medline

McDougle SD, Bond KM, Taylor JA (2015) Explicit and implicit processes constitute the fast and slow processes of sensorimotor learning. J Neurosci 35:9568-9579. CrossRef Medline

Medina JF, Garcia KS, Mauk MD (2001) A mechanism for savings in the cerebellum. J Neurosci 21:4081-4089. Medline

Miall RC, Jenkinson N, Kulkarni K (2004) Adaptation to rotated visual feedback: a re-examination of motor interference. Exp Brain Res 154: 201-210. CrossRef Medline

Oldfield RC (1971) The assessment and analysis of handedness: the Edinburgh Inventory. Neuropsychologia 9:97-113. CrossRef Medline
Orban de Xivry JJ, Lefèvre P (2015) Formation of model-free motor memories during motor adaptation depends on perturbation schedule. J Neurophysiol 113:2733-2741. CrossRef Medline

Osu R, Franklin DW, Kato H, Gomi H, Domen K, Yoshioka T, Kawato M (2002) Short- and long-term changes in joint co-contraction associated with motor learning as revealed from surface EMG. J Neurophysiol 88: 991-1004. Medline

Pearson TS, Krakauer JW, Mazzoni P (2010) Learning not to generalize: modular adaptation of visuomotor gain. J Neurophysiol 103:2938-2952. CrossRef Medline

Pessiglione M, Petrovic P, Daunizeau J, Palminteri S, Dolan RJ, Frith CD (2008) Subliminal instrumental conditioning demonstrated in the human brain. Neuron 59:561-567. CrossRef Medline

Redding GM, Wallace B (1993) Adaptive coordination and alignment of eye and hand. J Motor Behav 25:75-88. CrossRef Medline

Smith MA, Ghazizadeh A, Shadmehr R (2006) Interacting adaptive processes with different timescales underlie short-term motor learning. PLoS Biol 4:e179. CrossRef Medline

Taylor JA, Krakauer JW, Ivry RB (2014) Explicit and implicit contributions to learning in a sensorimotor adaptation task. J Neurosci 34:3023-3032. CrossRef Medline

Taylor JA, Ivry RB (2011) Flexible cognitive strategies during motor learning. PLoS Comp Biol 7:e1001096. CrossRef Medline

Wolpert DM, Ghahramani Z, Jordan MI (1995) An internal model for sensorimotor integration. Science 269:1880-1882. CrossRef Medline

Wolpert DM, Diedrichsen J, Flanagan JR (2011) Principles of sensorimotor learning. Nat Rev Neurosci 12:739-751. CrossRef Medline

Zarahn E, Weston GD, Liang J, Mazzoni P, Krakauer JW (2008) Explaining savings for visuomotor adaptation: linear time-invariant state-space models are not sufficient. J Neurophysiol 100:2537-2548. CrossRef Medline 